\title{
RETINAL PIGMENTATION DUE TO CHOROIDAL MELANO-SARCOMA \\ WITH OBSERVATIONS ON CONGENITAL GROUPED PIGMENTATION OF THE RETINA*
}

BY

\author{
ALEXANDER LINDSAY
}

Manitoba Clinic, Winnipeg

A FORM of grouped pigmentation of the retina may occur in association with detached retina due to choroidal melano-sarcoma and, when present, is probably diagnostic of this disease. Grouped pigmentation of the retina also occurs as a congenital stationary condition. Two cases of the former and three of the latter are presented below. Evidence is given that both forms of pigmentation may be due to deposition of pigment epithelial cells on the posterior surface of the retina from circulating subretinal fluid.

\section{Case Reports}

Case 1, a middle-aged man, attended Sunderland Eye Infirmary in December, 1946. The fundus of his right eye (Fig. 1a) showed a markedly swollen and enlarged optic disc. The appearance differed from that of papilloedema in that the physiological cup was clearly defined. A slightly grey nodular mass, with dark stippling of its lower part, protruded from the lower nasal quadrant of the disc. From the disc to the lower periphery of the fundus the retina was detached. No haemorrhages or exudates were visible. Pigment in the area of retinal detachment was distributed as follows:

(a) Group of eight large spots situated below the inferior temporal branches of the central retinal vessels ;

(b) T-shaped stippled area infero-temporal to the optic disc. The horizontal limb of the T was more dense than the vertical limb and had upturned ends. The vertical limb was of diminishing intensity and width from above downwards.

(c) Two stippled patches, one near the infero-medial margin of the optic disc and the other about one-and-a-half disc diameters below the disc.

(d) Small spots distributed along the peripheral branches of the retinal vessels.

(e) Irregularly scattered small spots in the lower periphery.

Two weeks later the eye was again examined and the various changes were seen to have taken place (Fig. 1b). The optic disc was more swollen. Nodular swellings had appeared all over the disc with the exception of a small temporal area and the region of the optic cup. The original swelling was larger and darker. Many striate and blotchy haemorrhages had appeared at the optic disc and in the detached part of the retina. The pigment changes consisted of disappearance of the large pigment spots (A), increase in extent and intensity of the stippled T-shaped area $(B)$, and some alteration in the position and size of the stippled patches $(\mathrm{C})$. The eye was excised.

Histological Examination.-A highly vascular mixed-cell melano-sarcoma of the choroid spread out deep to Bruch's membrane in the region immediately below the optic disc. Bruch's membrane was intact and the tumour had invaded the optic disc. 


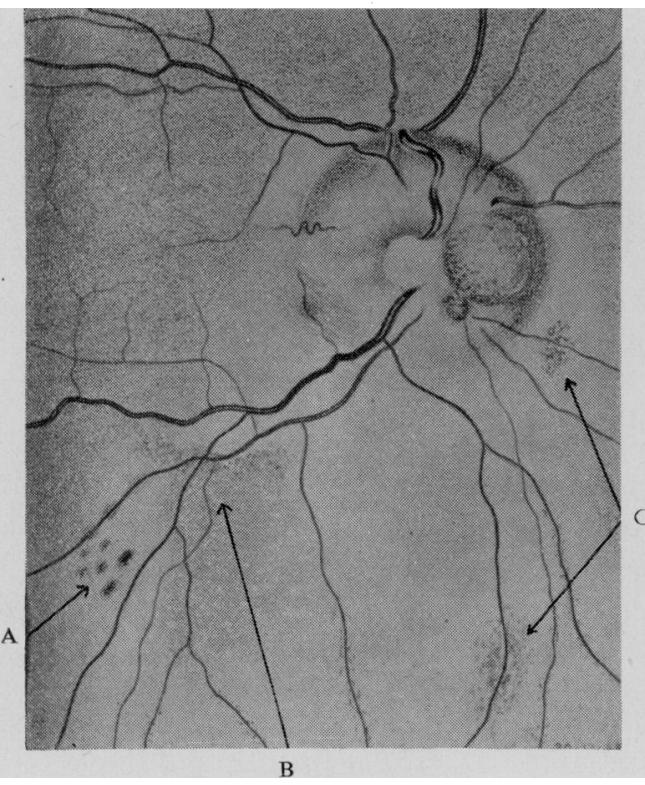

Fig. 1(a).-Juxta-papillary melano-sarcoma with detached retina showing pigmentation.

(A) Group of large pigment spots.

(B) T-shaped area of stippled pigmentation.

(C) Stippled patches.

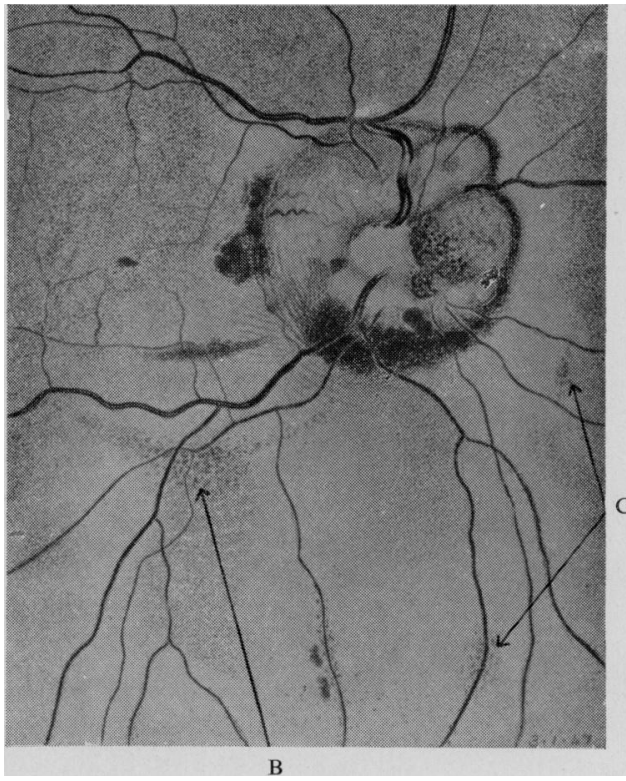

Fig. 1(b).-Same eye as Fig. 1(a) 2 weeks later, when the large pigment spots had disappeared.

(B) T-shaped area of pigmentation; more dense

and extensive than previously.

(C) Stippled patches.

Case 2, an elderly female, attended Glasgow Ophthalmic Institution in 1948 . Her right eye was blind with funnel detachment of the retina and markedly raised intra-ocular pressure. A dark mass was visible in the angle of the anterior chamber on the temporal side. Slit-lamp examination showed large golden-coloured pigment spots on the posterior retinal surface.

The eye was excised and a drawing made of the retina after removal of the cornea, iris, and lens (Fig. 2). The pigment was distributed as shown in the drawing and resembled,

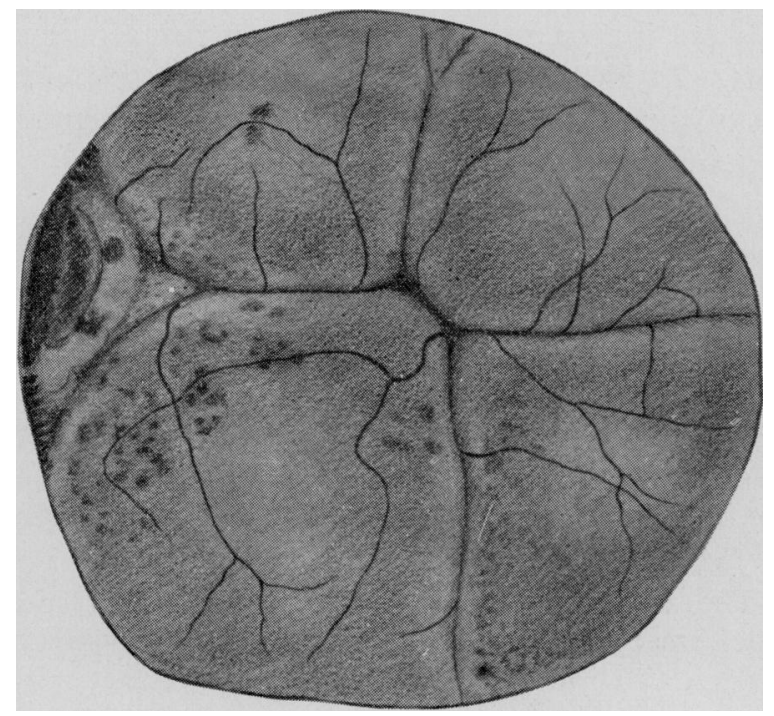

FIG. 2.-Retina of right eye in Case 2 after removal of cornea, iris, and lens, to show appearance and distribution of pigment spots. 
in appearance, the large pigment spots (A) in Case 1. It should be noted that the spots were situated at the upper and lower margins of the lateral retinal fold and at the nasal margin of the inferior fold.

Histological Examination.-A heavily pigmented mixed-cell melano-sarcoma involved the choroid, ciliary body, and iris on the temporal side. The tumour was entirely anterior to the equator. The pigment, previously seen with the slit-lamp, consisted of masses of pigment epithelial cells situated on the deep surface of the retina.

Comment on Cases 1 and 2.-Since Bruch's membrane was intact in Case 1, it is probable that the pigment spots $(a)$ were not composed of tumour cells. That they disappeared in a period of 2 weeks suggests that they were situated on the posterior surface of the retina rather than within the retinal tissue. In Case 2 the pigment spots consisted of clumps of pigment epithelial cells situated on the posterior surface of the retina. Probably the spots in both cases were similar except for their number and distribution.

The pigment distribution in Case 2 could have been due to deposition of pigment epithelial cells from the subretinal fluid in the presence of a convection current produced by a relatively high tumour temperature. The flow of subretinal fluid would be postero-medial in the upper half of the eye, hence the absence of pigment on the upper and medial retinal folds. In the lower half of the eye the flow would be antero-lateral with a tendency to deposit pigment on the nasal side of the lower retinal fold and the lower side of the lateral retinal fold. The pigment spots above the lateral fold would result from a lesser current in the upper outer quadrant due to less heated fluid gravitating before crossing the stalk of the detached retina.

The shape and behaviour of the T-shaped stippled pigment area (B) in Case 1 also suggests the occurrence of a subretinal convection current. The curved horizontal part of this area might represent the site of impingement upon the retina of suspended pigment epithelial cells which had ascended in front of the tumour; the vertical part might have resulted from the deposition of cells descending behind the retina. The intensity and extent of this form of pigmentation increased during the 2-week period of observation.

The following three cases of congenital grouped pigmentation of the retina attended the Manitoba Clinic, Winnipeg, during the past year. In each case the pigment spots were drawn, particular attention being paid to the distribution of the spots. In the drawings the retinal vessels were omitted and the distribution of the spots was outlined. All of the pigment was deep to the retinal blood vessels.

Case 3, a female aged 15, whose right eye showed pigment spots, arranged as shown in Fig. 3 (opposite). Two somewhat S-shaped bands of pigment spots, whose curves correspond in direction, extend peripherally from the optic disc.

Case 4, a female aged 15, in whose left eye the distribution of pigment was as shown in Fig. 4 (opposite). There are two incomplete S-shaped bands, the peripheral part of the band on the nasal side being double. The interrupted lines in the drawing indicate the probable outline of the missing parts.

Case 5, a female aged 10, with pigment spots in her left eye (Fig. 5, opposite). Some small pigment spots are present in the macular area and there are two bands of spots in the upper temporal quadrant of the fundus.

Comment on Cases 3 - 5.-The S-shaped arrangement of the pigment spots (Fig. 4) is probably not incidental, since it can also be seen in published drawings by 


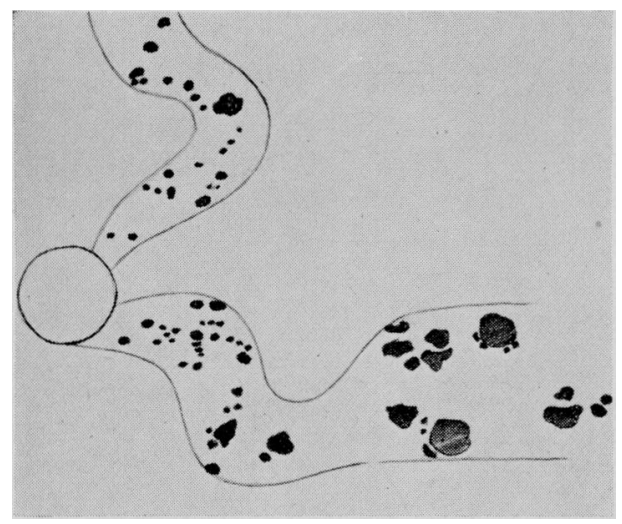

FIG. 3.-Congenital grouped pigmentation of retina, showing typical S-shaped distribution of spots, in two bands with corresponding curves.

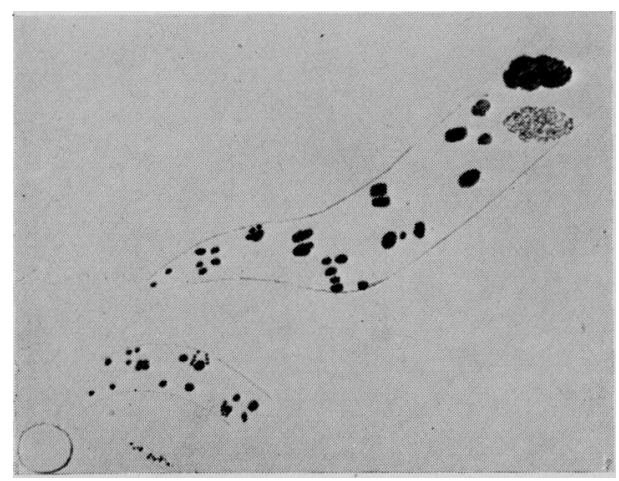

FIG. 5.-Congenital grouped pigmentation of retina, showing two short bands of spots in same meridian, and involvement of macular area.

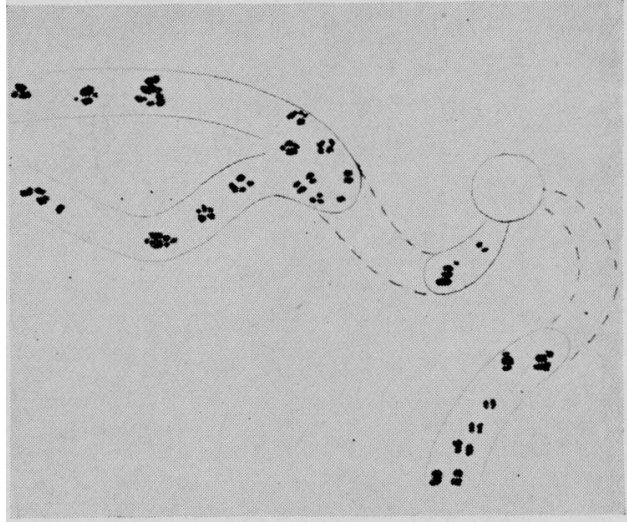

FIG. 4.-Congenital grouped pigmentation of retina, showing two incomplete S-shaped bands of pigment spots with branching of one peripherally.

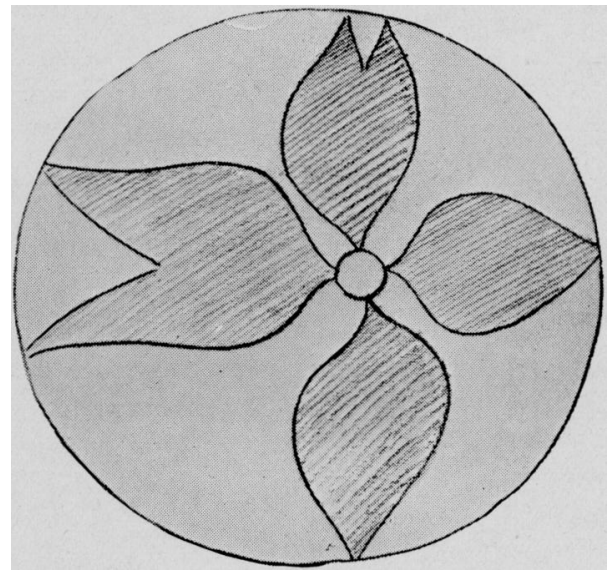

FIG. 6.-Shaded areas approximately represent parts of retina, before detachment, which would form folds in such a case as Case 2.

Höeg (1911), Holm (1921), Blake (1926), and Perera (1939), the last-mentioned showing three such brands with curves which correspond in direction.

The shaded areas in Fig. 6 are approximately those composing the retinal folds in Case 2 ; the margins of these areas resemble the S-shaped distribution of spots mentioned in the above cases of congenital grouped pigmentation. Corresponding margins have corresponding curves. During development, the retina may sometimes be sufficiently detached to form folds, free pigment cells in the subretinal fluid being deposited on them and giving rise to S-shaped bands of pigment spots which persist as congenital grouped pigmentation.

The branching of a retinal fold may account for the double nasal band of spots in Case 4. The arrangement of pigment in Case 5 suggests the occurrence of two short retinal folds, instead of one long one extending from the optic disc to the periphery of the fundus. 


\section{Discussion}

The often noted triangular sector distribution of congenital grouped pigmentation resembles the triangular distribution of keratic precipitates, which is due to deposition of cells from circulating fluid in the anterior chamber in cases of iridocyclitis.

Pigment spots may be distributed in groups radiating from the optic disc like rays from the sun. This distribution is seen in a drawing published by Kraupa (1917) and suggests that pigment-cells have been deposited on multiple radiating retinal folds.

The great thickness of the macular area between the 3rd and 7th foetal month may render it more resistant to folding, and may account for its being free of pigment spots in the great majority of cases. The characteristic congenital pigment groups, said to resemble the paw marks of an animal, are absent in cases of melano-sarcoma ; probably because they are formed by division of larger spots during growth of the eye.

I can give no reasons why circulation of subretinal fluid should occur; my main object has been to show that, in a number of cases, the appearances of congenital grouped pigmentation of the retina are such as might be expected to result from such a circulation.

\section{Summary}

(1) Two cases of retinal pigmentation due to choroidal melano-sarcoma and three cases of congenital grouped pigmentation of the retina are described.

(2) Reasons are given for thinking that both forms of pigmentation are due to deposition of pigment epithelial cells on the posterior surface of the retina from circulating subretinal fluid.

(3) The large pigment spots described in the two cases of choroidal melano-sarcoma are thought to be characteristic of this disease.

\section{REFERENCES}

Blake, E. M. (1926). Trans. Amer. ophthal. Soc., 24, 223 (Case 6).

Hötg, N. (1911!. Klin. Mbl. Augenheiik., 49 (1), 49 (Fig. 4).

Holm, E. (1921). Ibid., 67, 451 (Figs 1 and 2).

Kraupa, E. (1917). Arch. Augenheilk., 82, 67 (Fig. 7).

Perera, C. A. (1939). Arch. Ophthal. (Chicago), 21, 108. 